\title{
Synthesis and Verification of Biobased Terephthalic Acid from Furfural
}

\section{SUBJECT AREAS: \\ SUSTAINABILITY \\ METHODOLOGY}

\author{
Received \\ 4 December 2014 \\ Accepted \\ 9 January 2015 \\ Published \\ 4 February 2015
}

Correspondence and requests for materials should be addressed to K.K. (kkasuya@gunmau.ac.jp)

\author{
Yuya Tachibana ${ }^{1,2}$, Saori Kimura' \& Ken-ichi Kasuya'
}

'Division of Molecular Science, Faculty of Science and Technology, Gunma University, 1-5-1 Tenjin, Kiryu, Gunma 376-8515, Japan, ${ }^{2}$ PRESTO, Japan Science and Technology Agency (JST), 4-1-8 Honcho, Kawaguchi, Saitama 333-0012, Japan.

Exploiting biomass as an alternative to petrochemicals for the production of commodity plastics is vitally important if we are to become a more sustainable society. Here, we report a synthetic route for the production of terephthalic acid (TPA), the monomer of the widely used thermoplastic polymer poly(ethylene terephthalate) (PET), from the biomass-derived starting material furfural. Biobased furfural was oxidised and dehydrated to give maleic anhydride, which was further reacted with biobased furan to give its Diels-Alder (DA) adduct. The dehydration of the DA adduct gave phthalic anhydride, which was converted via phthalic acid and dipotassium phthalate to TPA. The biobased carbon content of the TPA was measured by accelerator mass spectroscopy and the TPA was found to be made of $100 \%$ biobased carbon.

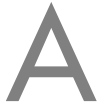

s social demand for renewable sources of commodity plastics has increased, biomass has attracted much attention as a promising alternative to petrochemicals ${ }^{1-3}$. The resources used to produce biobased plastics should be inedible, waste, and abundant. Furfural is an ideal biomass resource, as it is traditionally produced from cellulosic and waste biomass such as corncob, corn stock, and rice hull. Furthermore, it is extremely abundant, with a global output of 500,000-1,000,000 tonne/year ${ }^{4,5}$. The US Department of Energy (DOE) has stated that it is one of the most value-added chemicals derived from biomass ${ }^{6}$.

Another advantage of using furfural is that it can be readily converted to other useful chemicals due to its high reactivity ${ }^{7}$. Furfural is industrially converted to furan via decarboxylation and has been used as a source of biofuels ${ }^{7-10}$. In previous works, we have synthesised succinic acid and 1,4-butanediol from furfural, and polymerised them to the biodegradable thermoplastic resin poly(butylene succinate $)^{11-13}$. We have further used it to produce biobased acid anhydrides and polymerised them with diols to polyoxabicyclates, which are a potential alternative to commercially available transparent elastic polymers ${ }^{14}$.

In this work, we have focused on producing biobased poly(ethylene terephthalate) (PET). PET resin is a widely used commodity plastic with applications in fibre manufacture, packaging, and electric devices. Coca-Cola Ltd. and other beverage companies have adopted biobased-PET since 2009 when they announced that their containers would change from petroleum-based PET to biobased PET ${ }^{1,15,16}$. However, this commercially available biobased PET is composed of bio-ethylene glycol derived from bio-ethanol, and petroleum-based terephthalic acid (TPA) made from $p$-xylene produced by fractional distillation of naphtha. Consequently, its percentage biomass carbon content, as defined below, is a mere $20 \%$.

The development of a route to biobased TPA would allow the synthesis of fully biobased PET, an extremely attractive prospect from both economic and environmental viewpoints. Consequently, many researchers have attempted to develop a viable method to produce biobased TPA and its precursors. $p$-Xylene, a precursor of TPA, has been obtained from two different biomass resources: glyceride, supplied as a by-product of commercially available chemicals such as bio-diesel; and 5-hydroxymethyl-2-furfural, derived from cellulosic waste ${ }^{17}$. The monoterpene $p$-cymene has been directly oxidised to TPA ${ }^{18}$, and ethylene, which can be produced from bioethanol, has been converted to $p$-xylene ${ }^{19}$. The conversion of biomass chemicals such as ethylene, isobutene, furan, and dimethylfurfural to $p$-xylene using heterogeneous catalysts has also been investigated ${ }^{20-24}$. Furthermore, Toray industries Inc. launched the production of a fully biobased PET fibre with biobased TPA derived from isobutene in $2012^{25,26}$.

In this work, we propose a viable synthetic route, shown in Figure 1, for the preparation of biobased TPA from furfural alone, which is produced industrially from inedible cellulosic biomass. In addition, we verified that the TPA obtained was indeed biobased, as determined by the measurement of the biobased carbon content. Biobased carbon content has been adopted as a criterion for biomass-sourced chemicals in several international standards such as ISO, ASTM, and EN as it is the most practical and effective method for verifying that a material contains 


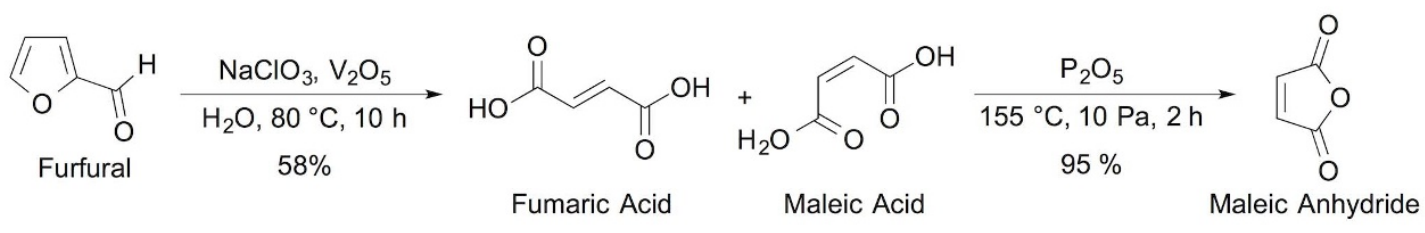

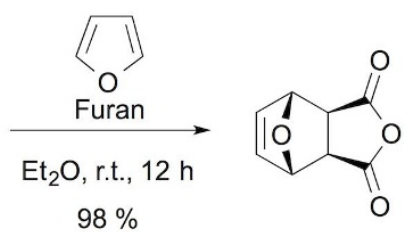

DA adduct

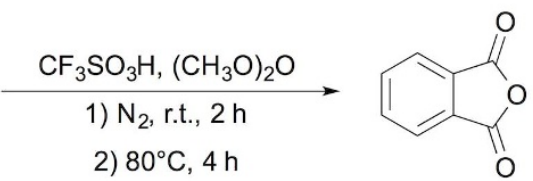

Phthalic Anhydride

$84 \%$

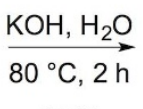

$98 \%$

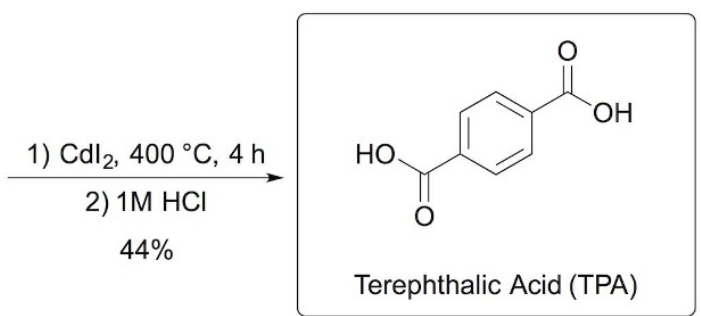

Figure 1 Synthetic route to biobased TPA from biomass-derived furfural. The furan used to produce the DA adduct is processed from the same biomass-derived furfural employed at the beginning of the route, making the product entirely biobased.

carbon derived from biomass ${ }^{27-29}$. It is determined by measuring the value of the ${ }^{14} \mathrm{C} /{ }^{12} \mathrm{C}$ ratio in the material, and is based on the assumption that this ratio for petroleum-derived (or 'ancient') carbon is 0 , while the ratio for biobased (or 'modern') carbon is $1 \times 10^{-12}$. In our work, this ratio was measured using accelerator mass spectroscopy (AMS) according to guidelines listed in ISO 16620-2. The details of the measurement procedure have been previously reported in the literature ${ }^{28}$.

\section{Results and Discussion}

Oxidation of furfural to fumaric acid and maleic acid. The oxidisation of furfural with $\mathrm{NaClO}_{4}$ as an oxidant, and $\mathrm{V}_{2} \mathrm{O}_{5}$ as a catalyst, gave a mixture of fumaric acid and maleic acid in $58 \%$ yield, lower than the $72 \%$ yield reported in the literature ${ }^{30}$. This lower yield could be caused by vigorous oxidation, which is difficult to control. The oxidation of furfural to maleic acid and fumaric acid has been performed by other means with improved yields and better selectivity for the products, either maleic acid or fumaric acid, elsewhere in the literature $e^{31,32}$. However, the oxidation with $\mathrm{NaClO}_{4}$ is, for our purposes, a more practical laboratory process, and, therefore, we adopted this traditional oxidation method in this study.

Dehydration of fumaric acid and maleic acid to maleic anhydride. The dehydration of the mixture of maleic acid and fumaric acid to maleic anhydride was performed using $\mathrm{P}_{2} \mathrm{O}_{5}$ as a dehydration agent. As the ratio of maleic acid and fumaric acid, determined by ${ }^{1} \mathrm{H}$ NMR, was $1: 7$, the yield of maleic anhydride could potentially be less than $30 \%$. However, once maleic acid was dehydrated to give maleic anhydride, phosphoric acid, produced by the reaction of $\mathrm{P}_{2} \mathrm{O}_{5}$ and water, could isomerise fumaric acid to maleic acid ${ }^{33}$. Consequently, the mixture of maleic acid and fumaric acid was quantitatively converted to maleic anhydride.

Diels-Alder (DA) reaction of anhydrous maleic acid and furan to the exo-DA adduct. The synthesis of furan from furfural could have been demonstrated in this study. However, commercially available furan is a biobased chemical and has been verified as such in previous work $^{13}$. The biobased carbon contents of the furan and furfural used in this study are shown below. Therefore, although we have not performed the process ourselves, for the purposes of this work, commercially available furan is assumed to be biobased.

The DA reaction of maleic anhydride and furan readily gave the DA adduct. At the beginning of the reaction, the regioselectivity of DA cyclisation is for the endo-adduct due to its kinetic stability. However, the DA reaction is reversible and, after some time, the product is converted to the more thermally stable exo-DA adduct ${ }^{34-36}$. Consequently, the reaction was carried out for $12 \mathrm{~h}$ at room temperature, yielding the DA adduct in almost quantitative yield. Melting point analysis showed the m.p. of the product to be $127-129^{\circ} \mathrm{C}$, corresponding to the exo adduct.

Dehydration of the exo-DA adduct to phthalic anhydride. The oxabicyclo moiety in the exo-DA adduct is readily dehydrated with an acid. We attempted to dehydrate the exo-DA adduct using sulfonic acid, phosphoric acid, and $\mathrm{P}_{2} \mathrm{O}_{5}$, but the yield and purity of the phthalic anhydride produced were not high enough to isolate it. However, a more effective protocol for the dehydration of the exoDA adduct has been recently developed in which it is treated with a mixture of trifluoromethane sulfonic acid and acetic anhydride ${ }^{37}$. We employed this new method and obtained phthalic anhydride in $84 \%$ yield.

Hydrolysis of phthalic anhydride to dipotassium phthalate. Phthalic acid was readily hydrolysed with aqueous potassium hydroxide to give dipotassium phthalate quantitatively.

Transfer reaction and acidification of dipotassium phthalate to TPA. Half a century ago, a transfer reaction known as the Henkel method, which converts dipotassium phthalate to dipotassium terephthalate at high temperature (above $400^{\circ} \mathrm{C}$ ) with $\mathrm{CdI}_{2}$ as a catalyst, was the most common industrial process for the production of TPA ${ }^{38,39}$. The development of an alternative method involving the oxidation of $p$-xylene to TPA led to the Henkel method losing its competitive 
advantage and falling out of favour. Consequently, it is rarely used industrially. However, in this study, we adopted the Henkel method to convert biobased phthalate to biobased TPA, as we found it to be a practical method for obtaining biobased TPA from furfural. The reaction was carried out with $\mathrm{CdI}_{2}$ at $420^{\circ} \mathrm{C}$, and the resulting mixture was acidified to give biobased TPA. At $44 \%$, the yield of TPA obtained in this study is not sufficient. However, this figure is obtained at the milligram scale, but the process was optimised industrially, so, therefore, it is reasonable to expect that the yield would increase for industrial production. Additionally, the Henkel method is proven as an industrial process, and, therefore, the commercial viability of this synthetic route from furfural to TPA is already established.

Biobased carbon content. The biobased carbon contents of the reagents and products are summarised in Table 1 . The synthesis of fully biobased TPA is verified by the fact that the values of furfural, furan, and TPA were almost $100 \%$. Thus, we can conclude that both the starting materials and product are fully biobased chemicals.

On the other hand, the values for furfural and furan reported previously were 100.8 and $105.0 \%{ }^{11,13}$ and slightly higher than those measured in this study. These values were obtained in 2010, while those in this study were obtained in 2014. Since the lot numbers of furfural and furan used in this study are different from those used in the previous study, the actual value of the ${ }^{14} \mathrm{C} /{ }^{12} \mathrm{C}$ ratio could be slightly different. This difference could be explained by the difference in the definition of biobased carbon content between ISO 16620-2 and ASTM D6866 and the manufacturing year of furfural and furan. In principle, the percentage of modern carbon (pMC) calculated from the ${ }^{14} \mathrm{C} /{ }^{12} \mathrm{C}$ concentration ratios, is the biobased carbon content. However, the pMC for biomass produced by fixation of $\mathrm{CO}_{2}$ in the atmosphere by photosynthesis was $108-110 \%$ in $2002^{27-29}$. The pMC is possibly slightly higher than $100 \%$ because of the continuing but diminishing effects of nuclear testing in the atmosphere in the 1950s, during which large amounts of ${ }^{14} \mathrm{C}$ were emitted into the atmosphere. Because the ${ }^{14} \mathrm{C}$ in all the samples is referenced to a "prebomb" standard, i.e., modern carbon-based oxalic acid radiocarbon [Standard reference material (SRM) 4990c, National Institute of Standards, USA], all pMC values must be multiplied by a cofactor to correct for the bomb carbon and to obtain the true biobased carbon content of the sample. In our previous study, the biobased carbon contents were calculated using a strong cofactor of 0.93 , that being the old value based on ASTM D6866 (2008) because the furfural and furan used were purchased before 2009. Nevertheless, the reason why the value of furan was above $105 \%$ is that it was produced before 2008 . In this study, as the reagents used were purchased in 2013, the biobased carbon contents were calculated using the new value for the weak cofactor of 0.95 based on ISO 16620-2. These indicate that the nuclear testing effect on the old reagents was strong and the biobased carbon content was above $100 \%$, even though the strong cofactor 0.93 was used, while the effect on the new reagents was weaker, giving a biobased carbon content of almost $100 \%$. Therefore, the biobased carbon contents of furan and furfural in this study were slightly different from the values measured in the previous study.
The precise method for the measurement of biobased carbon content is detailed in ISO 16620-2 and ASTM D6866 and is an industrially indispensable verification procedure ${ }^{29,40}$. It is important, not just to prevent mistakes by researchers, but also to detect whether supposedly biobased materials have undergone some contamination from, or carbon exchange with, petrochemical sources such as other reaction reagents or non-biobased solvents. For example, in the case of the Henkel method, the transfer reaction could involve the incorporation of a carbonyl carbon from carbon dioxide, produced as a by-product of a petrochemical process ${ }^{38,41}$. In addition, biobased carbon content measurement is also an invaluable method for identifying materials mistakenly or falsely supplied as biobased. Therefore, we propose that the measurement of biobased carbon content should be necessary when biobased chemicals are used, especially when the products can be synthesised from commercially available petroleum-derived starting materials or involve the use of non-biobased reagents or solvents.

In summary. We successfully synthesised biobased TPA from furfural and furan using viable and proven organic synthetic procedures. Furthermore, the biobased carbon content of the TPA that we synthesised confirmed that it is a truly biobased product. Using furfural as a single resource is a novel and interesting concept, since furfural can be produced from inedible cellulosic biomass.

The aim of this study was to propose a viable synthetic route from furfural alone to TPA, and we have succeeded in this. It is our hope that more research, conducted by both ourselves and, perhaps, other groups, will optimise this process so that it may be industrialised.

We finally propose that the measurement of biobased carbon content is indispensable as a verification method in the research area of biobased synthesis.

\section{Methods}

Materials. Furan, sodium chlorate, vanadium pentoxide, phosphorus pentoxide, methane sulfonic acid, acetic anhydride, cadmium iodide, toluene, and diethyl ether were purchased from Wako Pure Chemical Industries (Osaka, Japan). Furfural, potassium hydroxide, and hydrochloric acid were purchased from Kanto Kagaku Co., Inc (Tokyo, Japan). Phosphorus pentoxide was purchased from Kishida Chemical Co., Ltd (Osaka, Japan). Furfural, trifluoromethane sulfonic acid, and acetic anhydride were used after distillation under reduced pressure. All other chemicals were of reagent grade and used without further purification.

Instrumentation. ${ }^{1} \mathrm{H}$ NMR spectra were recorded on a $400 \mathrm{MHz}$ NMR spectrometer (JNM-ECX400; JEOL, Tokyo, Japan) using deuterated chloroform or deuterated dimethyl sulfoxide as a solvent, and tetramethylsilane as an internal standard.

Measurement of biobased carbon content ${ }^{28}$. Measurements of the ratios of the three carbon isotopes $\left({ }^{14} \mathrm{C},{ }^{13} \mathrm{C}\right.$, and $\left.{ }^{12} \mathrm{C}\right)$ using AMS were performed at the Institute of Accelerator Analysis Ltd (IAA) (Fukushima, Japan) using a 3-MV tandem accelerator (National Electrostatics Co., Middleton, WI, USA, Pelletron 9SDH-2). The pMC was calculated from the ${ }^{14} \mathrm{C} /{ }^{12} \mathrm{C}$ concentration ratios for the sample. The biobased carbon content was determined from the ratio of ${ }^{14} \mathrm{C} /{ }^{12} \mathrm{C}$ concentrations of the sample according to ISO $16620-2 . \Delta{ }^{14} \mathrm{C}$ is the isotope differential ratio of ${ }^{14} \mathrm{C}$ between the sample and reference material. Reference materials were also analysed using AMS. The biobased carbon content was calculated as follows:

$$
\begin{gathered}
{ }^{14} \mathrm{As}={ }^{14} \mathrm{C} /{ }^{12} \mathrm{C} \text { in sample } \\
{ }^{14} \mathrm{Ar}={ }^{14} \mathrm{C} /{ }^{12} \mathrm{C} \text { in reference material (NIST SRM 4990c) }
\end{gathered}
$$

\begin{tabular}{lccc} 
Table 1 | Biobased carbon content of biobased chemicals calculated from $\Delta{ }^{14} \mathrm{C}$ measured by accelerated mass spectrometry (AMS), in \\
accordance with ISO 16620-2 & $\Delta{ }^{14} \mathrm{C}$ \% & pMCa & biobased carbon content \% \\
Chemicals & 44.59 & 104.46 & 105.20 \\
\hline Furfural & 51.96 & 103.86 & 99.2 \\
Furan & 38.61 & 99.9 \\
Terephthalic Acid (TPA) &
\end{tabular}




$$
\Delta^{14} \mathrm{C}=\left[\left({ }^{14} \mathrm{As}^{14} \mathrm{Ar}\right) /{ }^{14} \mathrm{Ar}\right] \times 1000(\%)
$$

$$
\mathrm{pMC}=\Delta^{14} \mathrm{C} / 10+100(\%)
$$

Biomass carbon content $=0.95 \times \mathrm{pMC}(\%)$

Oxidation of furfural to fumaric acid and maleic acid $^{30}$. Furfural (36 g, $\left.367 \mathrm{mmol}\right)$ was carefully added dropwise to a solution of sodium chlorate $(80 \mathrm{~g}, 751 \mathrm{mmol})$ and vanadium pentoxide $(360 \mathrm{mg}, 1.98 \mathrm{mmol})$ in water $(10 \mathrm{~mL})$ at $90^{\circ} \mathrm{Cover} 3 \mathrm{~h}$, and the mixture was stirred at $80^{\circ} \mathrm{C}$ for a further $10 \mathrm{~h}$. The mixture was allowed to stand at room temperature for $11 \mathrm{~h}$, affording a white crystalline precipitate. The precipitate was filtered and dried to give $42.3 \mathrm{~g}(58 \%)$ of a mixture of fumaric acid and maleic acid as white crystals. ${ }^{1} \mathrm{H}$ NMR $\left(400 \mathrm{MHz}, \mathrm{DMSO}-d_{6}\right) \delta 6.61(2 \mathrm{H}, \mathrm{s},-\mathrm{CH}=$ of fumaric acid), $6.01(2 \mathrm{H}, \mathrm{s},-\mathrm{CH}=$ of maleic acid) $\mathrm{ppm}$.

Dehydration of fumaric acid and maleic acid to maleic anhydride ${ }^{42}$. The mixture of fumaric acid and maleic acid prepared above (3.00 g, $25.8 \mathrm{mmol})$ was mixed with diphosphorus pentoxide (5.00 g, $35.2 \mathrm{mmol})$ using a pestle and mortar. The resulting mixture was added to a sublimation apparatus and sublimated at $140^{\circ} \mathrm{C}$ and $0.4 \mathrm{kPa}$ for $3 \mathrm{~h}$. The resulting sublimate was collected to give $2.41 \mathrm{~g} \mathrm{(95 \% )} \mathrm{of} \mathrm{maleic}$ anhydride as white crystals. ${ }^{1} \mathrm{H}$ NMR $\left(400 \mathrm{MHz} \mathrm{CDCl}_{3}\right) \delta 7.46(2 \mathrm{H}, \mathrm{s}) \mathrm{ppm}$.

Diels-Alder (DA) reaction of anhydrous maleic acid and furan to exo-DA adduct $^{34}$. Maleic anhydride $(4.65 \mathrm{~g}, 47.4 \mathrm{mmol})$ was dissolved in diethyl ether $(50 \mathrm{~mL})$. Furan $(18 \mathrm{~mL}, 278 \mathrm{mmol})$ was added and the reaction mixture was allowed to stir at room temperature for $12 \mathrm{~h}$. The resulting precipitate was collected by filtration to give $7.77 \mathrm{~g}(98 \%)$ of white crystals. The melting point of white crystals was $127-129^{\circ} \mathrm{C}$. This indicates that the white crystals obtained is exo-DA adduct. ${ }^{1} \mathrm{H}$ NMR $\left(400 \mathrm{MHz}, \mathrm{DMSO}-d_{6}\right) \delta 6.58(2 \mathrm{H}, \mathrm{s}), 5.46(2 \mathrm{H}, \mathrm{s}), 3.18(2 \mathrm{H}, \mathrm{s}) \mathrm{ppm}$.

Dehydration of exo-DA adduct to phthalic anhydride ${ }^{37}$. The exo-DA adduct $(1.00 \mathrm{~g}, 6.02 \mathrm{mmol})$ was added to a mixture of methane sulfonic acid $(10.0 \mathrm{~mL}$, $154 \mathrm{mmol})$ and acetic anhydride $(2.0 \mathrm{~mL}, 21 \mathrm{mmol})$ under a $\mathrm{N}_{2}$ atmosphere at $0^{\circ} \mathrm{C}$. The reaction mixture was allowed to warm to room temperature and stirred for $2 \mathrm{~h}$. It was then heated to $80^{\circ} \mathrm{C}$ and stirred for $4 \mathrm{~h}$. After cooling to room temperature, the reaction mixture was extracted with toluene $(3 \times 20 \mathrm{~mL})$. The combined toluene extract was washed with saturated sodium hydrogen carbonate solution and saturated sodium chloride solution before being dried over anhydrous sodium sulfonate. After filtration, the organic layer was evaporated in vacuo to give $746 \mathrm{mg}(84 \%)$ of phthalic anhydride as white crystals. ${ }^{1} \mathrm{H}$ NMR $\left(400 \mathrm{MHz} \mathrm{CDCl}_{3}\right) \delta 8.08-7.95(2 \mathrm{H}, \mathrm{m}), 7.65-$ $7.52(2 \mathrm{H}, \mathrm{m}) \mathrm{ppm}$.

Hydrolysis to phthalic anhydride to dipotassium phthalate ${ }^{43}$. Phthalic anhydride $(500 \mathrm{mg}, 3.38 \mathrm{mmol})$ was dispersed in a solution of potassium hydroxide $(1.00 \mathrm{~g}$, $17.8 \mathrm{mmol})$ in water $(10 \mathrm{~mL})$. The reaction mixture was allowed to stir at $80^{\circ} \mathrm{C}$ for $2 \mathrm{~h}$. The resulting solution was poured into ethanol $(150 \mathrm{~mL})$ affording a white precipitate which was filtered to give $802 \mathrm{mg}(98 \%)$ of dipotassium phthalate as white crystals. ${ }^{1} \mathrm{H}$ NMR $\left(400 \mathrm{MHz}, \mathrm{D}_{2} \mathrm{O}\right) \delta 7.36-7.25(4 \mathrm{H}, \mathrm{m},-\mathrm{CH}=)$ ppm.

Conversion and acidification of dipotassium phthalate to TPA ${ }^{43}$. A mixture of dipotassium phthalate $(800 \mathrm{mg}, 3.30 \mathrm{mmol})$ and cadmium iodide $(40 \mathrm{mg}$, $0.11 \mathrm{mmol}$ ) was ground in a pestle and mortar. The mixture was placed in a sealing tube and sealed in vacuo. After heating to $420^{\circ} \mathrm{C}$ for $2 \mathrm{~h}$, the resulting solid was placed into hot water $(30 \mathrm{~mL})$. The dispersion was refluxed for $10 \mathrm{~min}$ and the remaining precipitate was removed by filtration. The filtrate was acidified with $1 \mathrm{M} \mathrm{HCl}$ and the resulting solid was filtered off to give $242 \mathrm{mg}(44 \%)$ of a white solid. ${ }^{1} \mathrm{H}$ NMR $\left(400 \mathrm{MHz}, \mathrm{CDCl}_{3}\right) \delta 8.03(4 \mathrm{H}, \mathrm{s},-\mathrm{CH}=) \mathrm{ppm}$.

1. Mülhaupt, R. Green polymer chemistry and bio-based plastics: dreams and reality. Macromol. Chem. Phys. 214, 159-174 (2013).

2. Queiroz, A. U. B. \& Collares-Queiroz, F. P. Innovation and industrial trends in bioplastics. Polym. Rev. 49, 65-78 (2009).

3. Chen, G.-Q. \& Patel, M. K. Plastics derived from biological sources: present and future: a technical and environmental review. Chem. Rev. 112, 2082-2099 (2012).

4. Hong, J., Zhang, Y., Xu, X. \& Li, X. Life cycle assessment of corn- and cassavabased ethylene production. Biomass and Bioenergy 67, 304-11 (2014).

5. Mao, L., Zhang, L., Gao, N. \& Li, A. FeCl 3 and acetic acid co-catalyzed hydrolysis of corncob for improving furfural production and lignin removal from residue. Bioresour. Technol. 123, 324-331 (2012).

6. Bozell, J. J. \& Petersen, G. R. Technology development for the production of biobased products from biorefinery carbohydrates - the US Department of Energy's 'Top 10' revisited. Green Chem. 12, 539-554 (2010).

7. Cai, C. M., Zhang, T., Kumar, R. \& Wyman, C. E. Integrated furfural production as a renewable fuel and chemical platform from lignocellulosic biomass. J. Chem. Technol. Biotechnol. 89, 2-10 (2014).

8. Brownlee, H. J. \& Miner, C. S. Industrial development of furfural. Ind. Eng. Chem. 40, 201-204 (1948).
9. Cass, O. W. Chemical intermediates from furfural. Ind. Eng. Chem. 40, 216-219 (1948).

10. Dutta, S., De, S., Saha, B. \& Alam, M. I. Advances in conversion of hemicellulosic biomass to furfural and upgrading to biofuels. Catal. Sci. Technol. 2, 2025 (2012).

11. Tachibana, Y., Masuda, T., Funabashi, M. \& Kunioka, M. Chemical synthesis of fully biomass-based poly(butylene succinate) from inedible-biomass-based furfural and evaluation of its biomass carbon ratio. Biomacromolecules 11, 2760-2765 (2010).

12. Kunioka, M., Masuda, T., Tachibana, Y., Funabashi, M. \& Oishi, A. Highly selective synthesis of biomass-based 1,4-butanediol monomer by alcoholysis of 1,4-diacetoxybutane derived from furan. Polym. Degrad. Stab. (2014) doi:10.1016/j.polymdegradstab.2014.05.011

13. Tachibana, Y., Masuda, T., Funabashi, M., Kasuya, K. \& Kunioka, M. Synthesis of biomass-based monomers from biomass-based furfural for polyesters and evaluation of their biomass carbon ratios. In Biobased Monomers, Polymers, and Materials, Smith, P. and Gross, R. Eds., ACS Symp. Ser., Press 1105, 91-110 (American Chemical Society 2012).

14. Tachibana, Y., Yamahata, M. \& Kasuya, K. Synthesis and characterization of renewable polyester containing oxabicyclic dicarboxylate derived from furfural. Green Chem. 15, 1318-1325 (2013).

15. Johansson, C. et al. Renewable fibers and bio-based materials for packaging applications - a review of recent developments. BioResources 7, 2506-2552 (2012).

16. Miller, S. A. Sustainable polymers: opportunities for the next decade. ACS Macro Lett. 2, 550-554 (2013).

17. Shiramizu, M. \& Toste, F. D. On the Diels-Alder approach to solely biomassderived polyethylene terephthalate (PET): conversion of 2,5-dimethylfuran and acrolein into p-xylene. Chem. Eur. J. 17, 12452-12457 (2011).

18. Colonna, M. et al. Synthesis and radiocarbon evidence of terephthalate polyesters completely prepared from renewable resources. Green Chem. 13, 2543-2549 (2011).

19. Lyons, T. W., Guironnet, D., Findlater, M. \& Brookhart, M. Synthesis of p-xylene from ethylene. J. Am. Chem. Soc. 134, 15708-15711 (2012).

20. Cheng, Y.-T., Wang, Z., Gilbert, C. J., Fan, W. \& Huber, G. W. Production of pxylene from biomass by catalytic fast pyrolysis using ZSM- 5 catalysts with reduced pore openings. Angew. Chem. Int. Ed. Engl. 51, 11097-11100 (2012)

21. Williams, C. L. et al. Cycloaddition of biomass-derived furans for catalytic production of renewable p-xylene. ACS Catal. 2, 935-939 (2012).

22. Do, P. T. M., McAtee, J. R., Watson, D. A. \& Lobo, R. F. Elucidation of Diels-Alder reaction network of 2,5-dimethylfuran and ethylene on HY zeolite catalyst. ACS Catal. 3, 41-46 (2013).

23. Lin, Z., Ierapetritou, M. \& Nikolakis, V. Aromatics from lignocellulosic biomass: economic analysis of the production of p-xylene from 5-hydroxymethylfurfural. AIChE J. 59, 2079-2087 (2013).

24. Agirrezabal-Telleria, I., Gandarias, I. \& Arias, P. L. Heterogeneous acid-catalysts for the production of furan-derived compounds (furfural and hydroxymethylfurfural) from renewable carbohydrates: a review. Catal. Today 234, 42-58 (2014).

25. Toray Industries, Inc (2012) Toray and Gevo Sign Bio-Paraxylene Offtake Agreement for the World's 1st Pilot-scale Fully Renewable, Bio-based Polyethylene Terephthalate (PET) Production. Available at http://www.toray.com/news/rd/ nr120627.html. Accessed October 7, 2014.

26. Yoshizawa, M. Toray's activities on bio-based polymers, Proceedings of Inno BioPlast 2013, Bangkok Thailand, 28 (2013).

27. Narayan, R. Biobased and biodegradable polymer materials: rationale, drivers, and technology exemplars. In Degradable Polymers and Materials. Khemani. K. and Schols C Eds., ACS Symp. Ser., Press 939, 282-306 (American Chemical Society 2006).

28. Funabashi, M., Ninomiya, F., Kunioka, M. \& Ohara, K. Biomass carbon ratio of biomass chemicals measured by accelerator mass spectrometry. Bull. Chem. Soc. Jpn. 82, 1538-1547 (2009).

29. Kunioka, M. et al. Biobased contents of natural rubber model compound and its separated constituents. Polymers (Basel). 6, 423-442 (2014).

30. Milas, N. A. Catalytic oxidations in aqueous solutions I. The oxidation of furfural. J. Am. Chem. Soc. 49, 2005-2011 (1927).

31. Guo, H. \& Yin, G. Catalytic aerobic oxidation of renewable furfural with phosphomolybdic acid catalyst: an alternative route to maleic acid. J. Phys. Chem. C 115, 17516-17522 (2011).

32. Choudhary, H., Nishimura, S. \& Ebitani, K. Highly efficient aqueous oxidation of furfural to succinic acid using reusable heterogeneous acid catalyst with hydrogen peroxide. Chem. Lett. 41, 409-411 (2012).

33. Terry, E. M. \& Eichelberger, L. Catalytic transmutation of maleic acid into fumaric acid. J. Am. Chem. Soc. 47, 1402-1412 (1925).

34. Schmart, I. M. \& Knot-Tso, M. E. Endo- vs. Exo-Selectivity in Diels-Alder reactions of maleic anhydride. J. Chem. Educ. 36, 81-82 (2004).

35. Sauer, J. Diels-Alder reactions II: the reaction mechanism. Angew. Chemie Int. Ed. 6, 16-33 (1967).

36. Sauer, J. \& Sustmann, R. Mechanistic aspects of Diels-Alder reactions: a critical survey. Angew. Chemie Int. Ed. 19, 779-807 (1980).

37. Mahmoud, E., Watson, D. A. \& Lobo, R. F. Renewable production of phthalic anhydride from biomass-derived furan and maleic anhydride. Green Chem. 16, 167 (2014). 
38. Raecke, B. Synthese von di- und tricarbonsäuren aromatischer ringsysteme durch verschiebung von carboxyl-gruppen. Angew. Chemie 70, 1-5 (1958).

39. Dozen, Y. Synthesis of aromatic dicarboxylic acids by Henkel and Cie process. J. Synth. Org. Chem. Japan 19, 570-581 (1961).

40. ISO 16620-2. Plastics -- biobased content -- part 2: determination of biobased carbon content. Available at: http://www.iso.org/iso/home/store/catalogue_tc/ catalogue_detail.htm? csnumber $=63767$. (Accessed: October 7, 2014)

41. Yoshiro, O. \& Masaru Hojo, M. M. Further studies on the preparation of terephthalic acid from phthalic or benzoic acid. J. Org. Chem. 25, 2082-2087 (1960)

42. Nystrom, R. F., Loo, Y. H. \& Leak, J. C. Synthesis of fumaric acid-2-C 14 and maleic anhydride-2-C 14 1. J. Am. Chem. Soc. 74, 3434-3435 (1952).

43. Raecke, B. \& Schirp, H. 2,6-Naphthalenedicarboxylic acid. Org. Synth. 40, 71 (1960).

\section{Acknowledgments}

This work, under the project 'Creation of essential technologies to utilise carbon dioxide as a resource through the enhancement of plant productivity and the exploitation of plant products,' was supported by the Precursory Research for Embryonic Science and

Technology (PRESTO) program of the Japan Science and Technology Agency (JST). We are thankful for valuable discussions with Masao Kunioka concerning biobased carbon content based on ISO 16620-2.

\section{Author contributions}

Y.T. and K.K designed this work and wrote the paper. Y.T. and S.K. carried out the synthetic experiments. All the authors participated in analysis and discussion of the results.

\section{Additional information}

Competing financial interests: The authors declare no competing financial interests.

How to cite this article: Tachibana, Y., Kimura, S. \& Kasuya, K.-i. Synthesis and Verification of Biobased Terephthalic Acid from Furfural. Sci. Rep. 5, 8249; DOI:10.1038/ srep08249 (2015).

This work is licensed under a Creative Commons Attribution 4.0 International License. The images or other third party material in this article are included in the article's Creative Commons license, unless indicated otherwise in the credit line; if the material is not included under the Creative Commons license, users will need to obtain permission from the license holder in order to reproduce the material. To view a copy of this license, visit http://creativecommons.org/licenses/by/4.0/ 